\title{
Philosophiques
}

\section{Concept de théorie et contexte diachronique}

\section{Jean Leroux}

Volume 5, numéro 2, octobre 1978

URI : https://id.erudit.org/iderudit/203099ar

DOI : https://doi.org/10.7202/203099ar

Aller au sommaire du numéro

Éditeur(s)

Société de philosophie du Québec

ISSN

0316-2923 (imprimé)

1492-1391 (numérique)

Découvrir la revue

Citer cet article

Leroux, J. (1978). Concept de théorie et contexte diachronique. Philosophiques, 5(2), 251-259. https://doi.org/10.7202/203099ar d'utilisation que vous pouvez consulter en ligne.

https://apropos.erudit.org/fr/usagers/politique-dutilisation/ 


\title{
CONCEPT DE THÉORIE ET CONTEXTE DIACHRONIQUE
}

\author{
par Jean Leroux
}

I. Le concept de théorie est certainement central en philosophie des sciences; cependant jusqu'à une date relativement récente, ce concept a été remarquablement peu élaboré par les milieux traditionnels en épistémologie des sciences. La tradition de l'empirisme logique nous a certes livré une problématique sophistiquée de la justification des théories scientifiques, ainsi qu'une discussion abondante sur la composition de leurs langages et le statut des soi-disant «termes théoriques », mais elle n'a pas pour autant problématisé en substance sa conception acceptée des théories empiriques. Celles-ci étaient fondamentalement envisagées comme des systèmes axiomatiques formels ou, en d'autres mots, comme des classes d'énoncés. Ce choix d'une conception assertive des théories ne saurait surprendre, si l'on se rend seulement présent à l'esprit la mesure dans laquelle l'empirisme logique véhiculait effectivement une philosophie particulière du langage.

On peut aussi, comme le fait Stegmüller ${ }^{1}$, voir dans cette fixation spécifique du concept de théorie l'influence de la recherche fondationnelle en mathématiques, filiation historique qui saurait encore moins surprendre. La métamathématique (au sens large du mot) considérait les théories mathématiques comme des systèmes cu des classes d'énoncés, concentrant ses recherches sur les relations logiques existant entre les éléments de ces classes. Obéissant à des critères méthodologiques dont la rigueur ne cédait en rien à ceux de la science qu'elle fondait, la métamathématique fut alors perçue comme un paradigme d'épistémologie fructueuse et servit de modèle

1. StecimLli.er, W., Theorie und Erfabrung. Zweites Halbband: Theorienstruktur und Theoriendynamik. Berlin, Springer 1973. p. 1-2. 
aux empiristes logiques; l'analyse de la syntaxe et de la sémantique logiques des langages empiriques devenait en quelque sorte leur moyen à eux de réaliser, en ce qui concerne les fondements des sciences empiriques, l'idée husserlienne d'une "Philosophie als strenge Wissenschaft ». Tout ce qui renvoyait aux dimensions pragmatiques de ces langages se voyait ainsi plus ou moins relégué au statut de "fait résiduel ", objet de sciences futures qui s'appelleraient psychoet socio-linguistique.

Cela évoque un autre rapprochement, cette fois-ci avec une science bien empirique, la linguistique justement. De même que de Saussure, se détachant des conceptions méthodologiques alors prévalentes, avait établi l'autonomie d'une discipline appelée linguistique synchronique, la fameuse distinction de Reichenbach entre le contexte de la découverte et le contexte de la justification des théories scientifiques se voyait érigée en principe d'autonomie d'une approche synchronique en philosophie des sciences (visant à la reconstruction logique des théories en tant que produits finis), approche autonome et préséante à l'étude des faits de diachronie en sciences (comme l'émergence, l'évolution, la supplantation des théories). Cette mise entre parenthèses des faits de diachronie au profit de l'élaboration d'une logique des sciences en contexte synchronique a sans doute elle aussi parrainé ce qui a été plus haut appelé la conception assertive (traduisant l'expression anglaise statement view) des théories scientitiques.

II. On sait que cette négligence des aspects pragmatiques et dynamiques de la science a été par après vivement mise en question, et que ce qui autrefois était conflit méthodologique entre inductivistes et déductivistes fait maintenant figure de querelle de famille, par suite des apports critiques provenant de milieux plutôt affiliés à l'histoire des sciences, ou, pour éviter la polysémie de cette expression, à l'historiographie des sciences. Des auteurs tels que Kuhn et Feyerabend, plus attentifs à la constitution historique des savoirs scientifiques qu'à leur reconstruction logique, ont pour ainsi dire sorti plusieurs épistémologues de leur sommeil dogmatique en indiquant justement ces dimensions négligées et en soutenant, exemples historiques à l'appui, que ce que la méthodologie 
traditionnelle avait pu dégager comme logique de la science demeurait toujours fuyant au niveau des pratiques scientifiques et, somme toute, diachroniquement inopérant.

Sur le conflit qui est alors apparu entre logique et histoire des sciences, et sur la controverse qui s'en est suivie entre les deux clans, Lakatos a semblé prendre une position mitoyenne en ce qu'il a accordé passablement d'attention à l'histoire des sciences dans l'élaboration de sa méthodologie des programmes de recherches scientifiques ${ }^{2}$. Reprenant la distinction entre histoire interne et histoire externe, il en est par ailleurs venu à identifier l'histoire interne des sciences à l'ensemble des faits qui se laissent reconstruire rationnellement (ce qui lui donnait en même temps l'occasion d'élever l'historiographie des sciences au statut de base empirique d'évaluation de toute épistémologie proposée) ; d'autre part, il identifiait l'histoire externe à tout ce qui ne se laisse pas récupérer méthodologiquement. On peut alors donner raison à Kuhn qui voit en cela une trivialisation renouvelée des méthodes d'approche historio-psychologiques et sociologiques ${ }^{3}$. Lakatos, le poppérien, aurait eu le même réflexe hautain à l'endroit de ces disciplines que jadis les empiristes logiques devant tout ce qui n'était pas syntaxe ou sémantique.

Sans pouvoir pour l'instant en dire plus long sur la question ouverte de savoir où exactement les considérations d'ordre typiquement métathéorique doivent faire place aux approches historio-psychologiques, ou sociologiques, j'aimerais ici retenir un seul point, qui est celui de la pertinence des méthodes logico-formelles pour l'élaboration d'une épistémologie diachronique des sciences - pour autant qu'une telle entreprise portera sur les produits des communautés scientifiques; allusion est faite en particulier aux méthodes de reconstruction des théories empiriques à la base de la sémantique logique, ou théorie des modèles, telles qu'on les retrouve

2. LAkatos, 1., "History of Science and its Rational Reconstructions ", in : Buck, R. C. ; Cohen, R. S. (eds.), Boston Studies in the Pbilosopby of Science VIII, PSA 1970. Boston, Reidel 1971. p. 105-8.

3. KuH, T. S., "Noies on Lakatos", in : Buck, R. C. ; Cohen, R. S. (eds), Boston Studies in the Pbilosopby of Science VIII, PSA 1970. Boston, Reidel 1971, p. 140)-1 
par exemple chez Przêęcki, Williams et Suszko ${ }^{4}$. Ceux qui sont hostiles à l'emploi de ces méthodes (ou à l'idée même de méthode) semblent fonder leur opinion sur le fait qu'elles sont inaptes à cerner, et encore moins à régler les vrais problèmes. Mais comme le fait remarquer Scheibe ${ }^{5}$, qui, pour un, reconnaît que ces méthodes font inévitablement violence aux théories-objets, tout dépend de ce que l'on veut bien considérer comme problème véritable. Certes, l'étude des facteurs économiques, sociaux, politiques et autres, qui agissent indubitablement sur la science et sur son évolution, ne saurait incomber au logicien. Mais il y a aussi certains facteurs internes de développement à l'œuvre en science - telles les notions de comparabilité des théories, de progrès théorique ou empirique - notions qui sont susceptibles d'une saisie sémantico-formelle. Toute épistémologie diachronique devra traiter de problématiques comportant certains aspects logiques - par exemple les notions de compatibilité, de contradiction et d'équivalence des théories - notions dont on ne pourra espérer aucune clarification sans le biais de précisions logiques préalables. Si l'on choisit de travailler à une méthodologie cohérente des sciences, où les approches synchronique et diachronique pourront être mises en rapport systématique, alors on veillera à ce que, d'une part, les concepts utilisés en contexte diachronique ne renoncent pas aux standards de rigueur atteints en épistémologie synchronique, et, d'autre part, à ce que les concepts utilisés en contexte synchronique soient évalués en fonction de leur opérationalité en épistémologie diachronique.

III. Si l'on applique ce dernier principe au concept de théorie scientifique en tant que classe d'énoncés, c'est-à-dire si on évalue la conception assertive en fonction de sa diachronisabilité, on obtient, par consensus, semble-t-il enfin, un verdict négatif. Une méthodologie fondée sur cette conception

4. PRrerEck1, M., The Logic of Empirical Theories. London, Routledge \& Kegan Paul 1969. WILliams, P. M., "On the Logical Relations between Expressions of Different Theories "The British Journal for the Philosopby of Science 24 (1973) 357-408. Stiszo, R., "Formal Logic and the Development of Knowledge ", in : Lakatos, I. ; Musgrave, A. (eds.), Problems in the Philosophy of Science. Amsterdam, North-Holland 1968. p. 210-22.

5. Schinke, E., "Vergleichbarkeit, Widerspruch und Erklärung ", in : Haller, R. : Göschl, J. (eds.), Philosophie und Physik. Braunschweig, Vieweg 1975. p. 58-9. 
achoppe lorsqu'il s'agit de rendre compte de l'immunité dont jouissent les théories scientifiques en période normale d'évolution, ou de fournir une base explicative adéquate pour les changements de théorie en période de révolution scientifique; plus spécifiquement, elle rencontre des difficultés face aux thèses de Duhem-Quine sur l'irréfutabilité empirique des théories scientifiques, et à celles de Kuhn-Feyerabend sur leur incommensurabilité. Et un supplément de sophistication démarcationiste n'y fera rien, si on en juge par les succès mitigés que rencontre la méthodologie des programmes de recherches scientifiques proposée par Lakatos. Bien que ce dernier fasse meilleur accueil aux composantes conventionalistes ou pragmatiques en science, et qu'il dilue la notion de rationalité en science jusqu'à l'état d'historisme, son approche ne parvient pas à surclasser ou même à atténuer les versions sceptiques des thèses d'irréfutabilité et d'incommensurabilité. Son plus grand mérite aura peut-être été de déplacer l'attention de la théorie vers la séquence des théories, séquence qui constitue maintenant l'unité minimale dont il est raisonnable (pour un démarcationiste) de se demander si elle est scientifique ou non. Sans préjuger des avantages d'un certain holisme accru, c'est-à-dire pour lequel l'unité porteuse de scientificité n'est plus le terme, ni l'énoncé, ni la théorie, mais la séquence des théories constituant un programme de recherche, j’aimerais revenir à ma préoccupation actuelle : exhiber un des concepts de théorie qui sont opérationnels en contexte diachronique comme en contexte synchronique, et jeter à cet effet un coup d'œil affable du côté de chez Kuhn et Feyerabend.

IV. La notion de théorie chez Feyerabend englobe, pour autant que je sache, les croyances ordinaires (comme la croyance en l'existence d'objets matériels), les mythes, les croyances religieuses, bref, tout point de vue suffisamment général pour constituer une "Weltanschauung " ${ }^{6}$. Une telle prodigalité au niveau d'un concept épistémologique central contrevient à la première partie du principe proposé à la fin du

6. Feyerabend, P. K., "Problems of Empiricism I ", in : Colodny, R. G. (ed.), Beyond the Edge of Certainty. Englewood Cliffs/NJ, Prentice Hall 1965. [University of Pittsburg Series in the Philosophy of Science, 2]. Note p. 219. 
chapitre II, principe auquel Feyerabend n'est évidemment pas tenu. Il faut cependant remarquer qu'une conception trop large des théories viendrait ni plus ni moins immuniser la thèse d'incommensurabilité, sinon la trivialiser. Mais ce qui apparaît être une qualité chez l'un est un défaut chez l'autre, et toute la discussion se voit reportée à un métaniveau qui déborde les positions que j'ai à prendre ici, à savoir qu'on a avantage à épargner certains domaines du principe de nonchalance (sloppiness) en matières sémantiques.

La notion de théorie chez Kuhn a eu elle aussi à subir au début des reproches d'ambiguiité. Kuhn a apporté à son concept de paradigme des modifications ultérieures importantes, en introduisant la notion de matrice disciplinaire en tant qu'objet d'allégeance commune et principe d'individuation des communautés scientifiques. Une matrice disciplinaire comporte 1 . des généralisations symboliques (comme $f=m \cdot a), 2$. des modèles (l'atomisme, par exemple), 3. des valeurs (simplicité, précision, etc . . .) et 4 . des exemplaires, c'est-à-dire des cas exemplaires d'énigmes résolues, des cas typiques d'application réussie de la théorie ${ }^{7}$. Si l'on fait une distinction entre cette dernière composante matérielle (où le domaine d'application de la théorie est paradigmatiquement déterminé) et les trois premières composantes (d'ordre plutôt conceptuel), on obtient la représentation kuhnienne d'une théorie en tant que couple $\langle\mathrm{K}, \mathrm{I}\rangle$, où, reprenant une analogie familière aux lecteurs de Quine, $\mathrm{K}$, la composante conceptuelle, est l'élément nucléaire, et I, la composante matérielle, est l'élément périphérique. L'activité scientifique en période normale vise à l'aménagement de la périphérie sans réaménagement essentiel du noyau, alors qu'en période de crise et de révolution, c'est le noyau qui est mis en question et remplacé. Kuhn en venait récemment à caractériser $\mathrm{K}$ et $\mathrm{I}$ comme un domaine de connaissance et un domaine de croyance, respectivement.

V. Ces dernières indications de Kuhn pointent en direction

\footnotetext{
KLHN, T. S., "Postscript - 1969 ", in : $2^{\mathrm{e}}$ ed. augm. The Structwe of Scientific Retolutions. Chicago, University Press 1970. 2e partie. Aussi : "Reflections on my Critics", in: Lakatos, I. ; Musgrave, A. (eds.), Criticism and the Growth of Knouledge. Cambridge, University Press 1970. p. 271-4
} 
d'un mouvement de pragmatisation accrue en matières épistémologiques, particulièrement là où il est dit que l'emploi de théories scientifiques en période normale a pour effet de circonscrire un domaine de croyance. William Harper ${ }^{8}$ a récemment proposé une approche méthodologique qui va sans détours dans cette direction, en ce sens qu'il y a pragmatisation du concept formel de théories scientifiques; celles-ci y sont considérées comme des classes d'énoncés de croyance (belief sentences). Délaissant ainsi la conception assertive, Harper rode les méthodes d'approche développées par la pragmatique formelle et il parvient, semble-t-il, à atténuer les versions sceptiques de la thèse d'incommensurabilité.

Joseph Sneed ${ }^{9}$ a également proposé une méthode de reconstruction logique des théories physiques sur la base d'une conception non assertive. Son approche lui permet de bien reconstituer dans leurs principaux traits formels les idées kuhniennes sur le développement d'une science en période normale, et aussi de désarmer les reproches d'irrationalisme souvent formulés à leur endroit. Sneed reconstruit lui aussi les théories physiques comme couple $\langle\mathrm{S}, \mathrm{I}\rangle$, où $\mathrm{S}$ et I sont respectivement des composantes formelle et matérielle; plus spécifiquement, $\mathrm{S}$ représente une structure mathématique et I des systèmes physiques. La théorie nous sert à affirmer que ces domaines quelconques d'obiets possèdent telle et telle structure mathématique ; c'est ainsi que l'on peut se représenter grosso modo les assertions empiriques de la théorie. La structure mathématique en question est caractérisée par un prédicat ensembliste, tel que décrit par le système d'axiomes de la théorie physique; Sneed prend ici exemple sur la méthode d'axiomatisation informelle de la mécanique classique des particules, telle qu'exposée par Suppes ${ }^{10}$, c'est-à-dire par définition d'un prédicat ensembliste. Cette théorie physique peut donc être reconstituée dans sa formulation axiomatique sous la forme du prédicat « est une mécanique classique des

8. HARPER, W. L., "Rational Conceptual Change " (à paraître).

9. SNEED, J. D., The Logical Structure of Matbematical Pbysics. Dordrecht/Holland, Reide! 1971.

10. M.Kinsey, J. C. C. ; Sligar, A. C. ; Sluppes, P., "Axiomatic Foundations of Classical Particle Mechanics ". Journal of Rational Mechanics and Analysis II (1953) 253-72. 
particules ». On a donc l'idée d'un prédicat qui, d'une part, axiomatise informellement la théorie, et, d'autre part, (lorsqu'il s'agit de considérer les assertions empiriques de la théorie), est plus ou moins heureusement applicable à un ensemble de systèmes physiques, tout en possédant, bien sûr, des instances typiques d'applicabilité effective - comme, par exemple dans ce cas-ci, le système solaire, ou encore les corps à l'intérieur du champ de gravité terrestre. Représenter ainsi une théorie physique comme une structure formelle cum domaine d'applications revient à considérer cette première non plus comme une classe d'énoncés, mais plutôt comme quelque chose qui ne procure des énoncés que lorsque appliqué à des domaines quelconques d'objets. L'idée maîtresse demeure que l'on se sert d'une théorie comme d'un prédicat pour parvenir à des assertions empiriques; si l'on veut que ce prédicat tienne lieu de "schème conceptuel ", on dira qu'il renvoie à la notion kuhnienne de vision du monde relative à toute théorie, ou, encore, à la notion kantienne de structures formelles qui seraient des conditions de possibilité de l'expérience.

L'approche de Sneed tire son diachronisme du fait que l'on peut distinguer dans $\mathrm{S}$ un noyau $\mathrm{N}$, qui se retrouve dans les multiples formulations de la théorie et qui en exprime la loi fondamentale (par exemple la deuxième loi de Newton en mécanique classique des particules), et des extensions théoriques $\mathrm{E}$ de ce noyau (provenant de la formulation de lois additionnelles, comme par exemple ici la loi de la gravitation ou la loi de Hooke). À cette distinction à l'intérieur de la composante formelle s'en ajoute une autre à l'intérieur de la composante matérielle : du domaine d'applications visées I de la théorie on distingue un domaine d'applications typiques Io, sous-ensemble de I paradigmatiquement déterminé et affilié à $\mathrm{N}$. Comme le fait bien remarquer Diederich ${ }^{1}$, on obtient par là un modèle qui représente un explicat de la notion kuhnienne de science normale: un modèle de progrès théorique, qui consiste à maximaliser E par rapport à I (c'est-à-dire à porter des assertions de plus en plus spécifiques sur un domaine donné), et un modèle de progrès empirique, qui consiste à

11. DIEDERICH, W. (ed.), Theorien der Wissenschaftsgeschichte. Frankfurt/Main, Suhrkamp 1974. [Theorie-Diskussion]. p. 31-2. 
maximaliser I par rapport à E (c'est-à-dire la recherche de nouvelles instances d'applicabilité). La thèse d'irréfutabilité empirique ou d'immunisation de la théorie devant les faits récalcitrants s'explique donc par le jeu «normal » inter-reliant l'extension du noyau et l'augmentation du domaine visé d'application, processus ne laissant aucune prise à quelque principe de falsification que ce soit. Quant à la thèse d'incommensurabilité, elle renvoie à la disparité possible de deux noyaux structurels, sans pour cela empêcher radicalement la comparabilité de leurs pouvoirs respectifs de systématisation des champs de connaissance, et a fortiori l'idée de progrès postrévolutionnaire. Il semble cependant que le modèle sneedien rencontre plus de difficultés pour reconstruire rationnellement les idées de Kuhn sur la "science révolutionnaire"; pour ce faire, il faudrait commencer à en dire plus long sur $\mathrm{I}^{12}$.

VI. Ces deux approches, l'une mentionnée et l'autre sommairement décrite, ont donc ceci en commun qu'elles se départissent de la conception des théories scientifiques en tant qu'entités linguistiques. Cela est nécessaire puisque l'étude des relations interthéoriques en science (comme d'ailleurs déjà la problématique typiquement synchronique des relations théorie-expérience) ne saurait se faire sur un plan purement linguistique, mais bien sur un plan sémantico-pragmatique. Il me semble évident que l'on a avantage, en épistémologie, à exploiter plus avant les techniques de la pragmatique et de la sémantique formelles là où leur emploi n'est pas indu ; et il m'est avis qu'il existe effectivement de tels domaines, même en contexte diachronique, 'même' voulant marquer ici un léger euphémisme.

Université de Montréal

12. D. Shapere, pour un, va dans cette direction. Cf. «Scientific Theories and their Domains ", in : Suppe, F. (ed.), The Structure of Scientific Theories. Urbana, University of Illinois Press (2céd. augm.) 1977. p. 518-65. 